\title{
A Registration Approach to Endoscopic Laser Speckle Contrast Imaging for Intrauterine Visualisation of Placental Vessels
}

\author{
Gustavo Sato dos Santos ${ }^{1,2}$, Efthymios Maneas ${ }^{1,2}$, Daniil Nikitichev², \\ Anamaria Barburas ${ }^{2}$, Anna L. David ${ }^{3}$, Jan Deprest ${ }^{4}$, Adrien Desjardins ${ }^{2}$, Tom \\ Vercauteren $^{1,2}$, and Sebastien Ourselin ${ }^{1,2}$ \\ 1 Translational Imaging Group, CMIC, University College London, NW1 2HE, UK \\ 2 Dept. Med. Phys. and Biomed. Eng., University College London, WC1E 6BT, UK \\ 3 Institute for Women's Health, University College London, WC1E 6HX, UK \\ 4 Dept. of Obstetrics, University Hospitals KU Leuven, Belgium
}

\begin{abstract}
Intrauterine interventions such as twin-to-twin transfusion syndrome procedure require accurate mapping of the fetal placental vasculature to ensure complete photocoagulation of vascular anastomoses. However, surgeons are currently limited to fetoscopy and external ultrasound imaging, which are unable to accurately identify all vessels especially those that are narrow and at the periphery. Laser speckle contrast imaging (LSCI) is an optical method for imaging blood flow that is emerging as an intraoperative tool for neurosurgery. Here we explore the application of LSCI to minimally invasive fetal surgery, with an endoscopic LSCI system based on a 2.7-mm-diameter fetoscope. We establish using an optical phantom that it can image flow in 1-mm-diameter vessels as far as $4 \mathrm{~mm}$ below the surface. We demonstrate that a spatiotemporal algorithm produces the clearest images of vessels within $200 \mathrm{~ms}$, and that speckle contrast images can be accurately registered using groupwise registration to correct for significant motion of target or probe. When tested on a perfused term ex vivo human placenta, our endoscopic LSCI system revealed small capillaries not evident in the fetoscopic images.
\end{abstract}

\section{Introduction}

An augmented view of vasculature and blood flow is required for surgical procedures that rely on accurate identification of the vessels. Laser speckle contrast imaging (LSCI) is emerging as an intraoperative tool for monitoring tissue perfusion and blood flow, particularly in neurosurgery where its clinical potential has been recently demonstrated [1]. Despite its potential for imaging perfusion and blood flow in minimally invasive procedures, relatively few applications of LSCI for endoscopic surgery have been described [2]. The present study proposes a novel application for endoscopic LSCI for intrauterine fetal surgery.

In twin-to-twin transfusion syndrome (TTTS) therapy, the surgeon aims to photocoagulate anastomosing placental vessels that cause a net transfusion of 
blood between identical twins sharing a placenta [3]. Success depends on the degree of remaining anastomoses, since perinatal morbidity and mortality is highest in cases where anastomoses are not identified or completely photocoagulated [4]. Narrow vessels $(<2 \mathrm{~mm})$ are most likely to be missed or incompletely photocoagulated. Endoscopic LSCI can potentially help the surgeon identify smaller and less accessible vessels that are difficult to see in standard fetoscopy images.

Due to its nature, LSCI is highly sensitive to motion of the camera relative to tissue. This sensitivity is of particular concern in endoscopic surgeries when the camera is often handheld and the tissue can also move substantially. We aim to address this issue in two complementary ways: (1) by generating the clearest images within a short acquisition interval; and (2) by compensating for motion over longer intervals. For the first way, we evaluate three methods for estimating speckle statistics from data acquired in $200 \mathrm{~ms}$ to determine which yields the largest contrast between vasculature and background. And for the second way we evaluate two approaches for registering speckle contrast images in the presence of motion between the endoscope and the anatomy, and demonstrate that groupwise registration is preferable to a previously proposed method $[5,6]$. We tested our approach on an optical phantom and also on a perfused ex vivo human placenta, and in both cases tested the method's robustness to motion.

\section{Methods}

Endoscopic LSCI. A typical LSCI paradigm involves illumination of tissue with a spatially and temporally coherent laser source and reception of light with a camera. Our approach for endoscopic LSCI comprised a high-resolution sCMOS camera (ORCA-Flash4.0v2, Hamamatsu, Japan; pixel size: $6.5 \mu \mathrm{m}$ ) with highmagnification zoom lens (MVL6X12Z, Thorlabs, Germany), a 2.7-mm diameter fetoscope (Hopkins II, Karl Storz, Germany), and a $785 \mathrm{~nm}, 450 \mathrm{~mW}$ fibrecoupled laser (BWF1, B\&W Tek, USA). The fibre-coupled laser was positioned to create a specular illumination on the target area, and images were acquired using the sCMOS camera placed at the proximal end of the fetoscope (Fig. 1(a)). In all experiments, the sCMOS sensor's exposure time was set to $5 \mathrm{~ms}$ and frames were acquired at $100 \mathrm{fps}$ and at full resolution (2048x2048).

Speckle contrast estimation. When coherent light reflects off an irregular surface such as tissue, it forms a speckle pattern due to random interference. LSCI is based on the principle that the rate of change of speckle intensity is a function of the average velocity of the tissue particles that reflect light [7]. Speckles depend on the movement of scatterers and the sensor exposure time; by measuring the statistics of speckle intensities, it is possible to obtain highresolution images of the vasculature and also relative estimates of blood flow [1]. Speckle contrast images are computed from raw images by taking the ratio of the standard deviation to the mean pixel intensity: $K=\sigma / \mu$. There are three algorithms for estimating speckle contrast: (1) temporal, by computing the statistics of each pixel across time; (2) spatial, by computing the statistics within a square 
window around each pixel; and (3) spatiotemporal, by computing the statistics in a spatiotemporal cube window around each pixel. To evaluate these algorithms, we generate speckle contrast images from stacks of 20 raw images using each algorithm and compare the resulting images. For the spatial algorithm, we use a $14 \mathrm{x} 14$ kernel (typical kernel size: $0.2 \mathrm{~mm}$ ) to compute the spatial statistics and take the average of the 20 spatial contrast images to obtain a single image. A $14 \times 14 \times 20$ kernel is used for the spatiotemporal algorithm.

Registration of speckle contrast images. We compared two strategies for registering speckle contrast images to compensate for motion during image acquisition. The first one was proposed in $[5,6]$, and uses the image at the start of acquisition as a fixed reference for the registration of all other images. We propose an alternative strategy: to use groupwise registration and register all images to their average image, which is iteratively refined after each round of registrations. This approach was adapted from [8], except that affine transformations are applied on 2D images. Groupwise registration starts by taking the average of all unregistered images and using it as the initial fixed image; after registering all images relative to it, the fixed image is recomputed as the average of the registered images, and this procedure is repeated. We used a total of 5 iterations to register 20 images in the moving phantom experiments, and 10 iterations to register 100 images in the placenta experiment. Both registration strategies described above used the same spatial speckle contrast images and the same number of iterations. We used Elastix to perform the registrations [9], and in all cases used the same set of registration parameters: affine transform as the transformation type, mutual information as the similarity measure, and adaptive stochastic gradient descent as the optimisation procedure.

Experimental setup. An optical phantom was used to evaluate the ability to image flow at different depths using our endoscopic LSCI approach. The phantom was made using a 3D-printed mould comprising two parts: a $13 \times 26$ $\mathrm{mm}$ rectangular chamber with $1 \mathrm{~mm}$ holes on the side, and a plate with 1-mm diameter rods spaced $2 \mathrm{~mm}$ horizontally and $1 \mathrm{~mm}$ vertically (Fig. 2(a)). The mould was filled with $5.5 \%$ agar solution mixed with $0.5 \%$ intralipid (SigmaAldrich, USA), and the rods were removed after the agar had set to create wall-less vessels (i.e., grooves in the agar). Speckle images were acquired as the phantom vessels were perfused with $1 \%$ intralipid at a constant rate $(60 \mathrm{~mL} / \mathrm{h})$ using a syringe driver (Alaris, CareFusion, USA). Intralipid has similar optical properties as biological tissue and, at $1 \%$ concentration, it has been used as blood phantom in LSCI studies. For the moving phantom experiments, the phantom was mounted on a motorised linear stage (MT1-Z8, Thorlabs, Germany) and moved at a constant speed of $4 \mathrm{~mm} / \mathrm{s}$ or $2 \mathrm{~mm} / \mathrm{s}$ longitudinally to the imaging plane. The working distance between fetoscope tip and phantom was $1.3 \mathrm{~cm}$ or $2.5 \mathrm{~cm}$. Constant intralipid flow was maintained in the 2 -mm-deep vessel.

Speckle contrast images were evaluated by computing the average signal-tonoise ratio (SNR) of the pixel contrast values in the perfused vessel region. ROI 
and background region were defined for each vessel (Fig. 2(b)), and the SNR was computed as: $S N R=\left|\mu_{R O I}-\mu_{b g}\right| / \sigma_{b g}$, where $\mu_{R O I}$ is the average contrast value in the ROI, and $\mu_{b g}$ and $\sigma_{b g}$ are the average and standard deviation of the contrast values in the background, respectively. Different selections for the background region were tested, with similar results.

For testing on ex vivo placenta, a term placenta was collected at UCLH following a C-section delivery and after obtaining a written informed consent from the mother (all procedures were approved by UCLH Ethics Committee). The placenta was placed on a flat surface and one of its surface vessels was perfused with $1 \%$ intralipid at $30 \mathrm{~mL} / \mathrm{h}$. The perfused vessel was imaged downstream from the cannula with our endoscopic LSCI system (Fig. 1(b)). To simulate handheld movement of the fetoscope, the distal end of the fetoscope was tapped and made to wobble by $<1 \mathrm{~cm}$ over $1 \mathrm{~s}$ during image acquisition.

\section{$3 \quad$ Experiments and results}

Endoscopic LSCI of a perfused placenta. The endoscopic LSCI system was initially tested on a perfused ex vivo placenta (Fig.1(b)). A 1-mm-diameter placental vessel was cannulated and continuously perfused with $1 \%$ intralipid solution, and the fetoscope was positioned to image the perfused vessel (Fig. 1(c)). 100 raw speckle images were acquired, and the estimated spatiotemporal speckle contrast image revealed the perfused vessel in high contrast relative to background (average pixel SNR $=3.28$; Fig. 1(e)). Furthermore, a branching capillary was clearly visible in the speckle contrast image (Fig. 1(e)), but was not as evident under white light illumination (Fig. 1(b) and Fig. 1(c)).

Evaluation of registration-free speckle contrast estimation methods in the absence of confounding motion. Three algorithms for estimating speckle contrast were tested on a perfused optical phantom (Fig.2(a) and Fig. 2(b)). Vessels at 1,2,3 and 4mm depth were perfused one at a time, and the phantom was placed at 1 or $2 \mathrm{~cm}$ from the fetoscope in air, or $1 \mathrm{~cm}$ in water. Intralipid flow could be reliably imaged down to $4 \mathrm{~mm}$ depth in air, or $3 \mathrm{~mm}$ depth in water (Fig. 2(c) and Fig. 2(d)). SNR analysis indicated that the spatiotemporal method consistently produced the highest contrast for intralipid flow.

Evaluation of registration algorithms on a moving phantom. The phantom was placed on a linear stage and moved at $4 \mathrm{~mm} / \mathrm{s}$ or $2 \mathrm{~mm} / \mathrm{s}$ longitudinally to the image plane (Fig. 3(a)) with the fetoscope at a fixed position and orientation. 20 frames of raw speckle image were acquired during movement, and the resulting 20 spatial speckle contrast images were registered, or spatially normalised, to estimate motion. Groupwise registration recovered the linear translation in the $\mathrm{X}$ direction with the phantom at $1.3 \mathrm{~cm}$ or $2.5 \mathrm{~cm}$ distance, whereas registering to the first frame failed in both accounts (Fig. 3(b), Fig. 3(c)). Speckle contrast images were clearest with a combination of groupwise registration and spatiotemporal contrast estimation (Fig. 3(e)). 

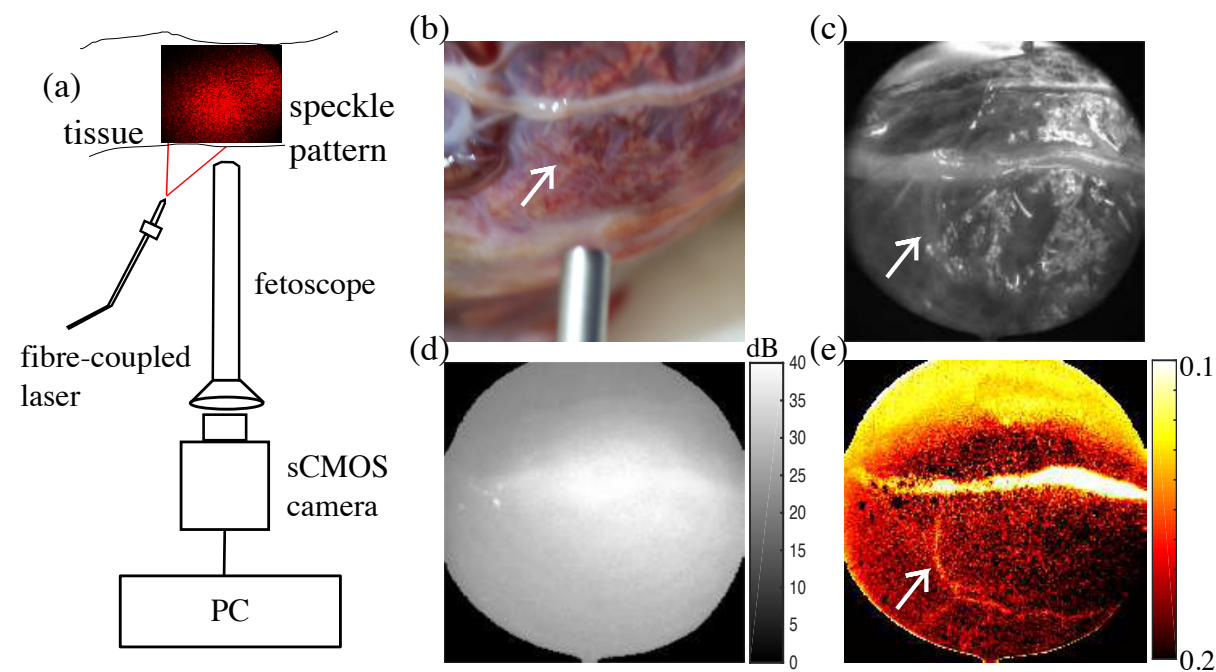

Fig. 1. (a) Diagram of the endoscopic LSCI system. (b) A photograph showing the tip of the fetoscope and the area on the placenta being imaged. The vessel at the image top is $1 \mathrm{~mm}$ in diameter and was continuously perfused with $1 \%$ intralipid. (c) Fetoscopic image acquired under white light illumination. (d) Raw fetoscopic speckle image acquired using laser illumination. (e) Speckle contrast image showing perfused vessel and a branching capillary; lower contrast values (warmer colours) indicate flow. The white arrows in (b), (c) and (e) indicate the capillary's location in each image.

Evaluation of groupwise registration on perfused placenta. While imaging the same region of the placenta as in Fig. 1 the tip of the fetoscope was tapped, making it wobble vertically. The spatiotemporal contrast image of the data acquired during motion contains dark artefacts in the background (Fig. $4(\mathrm{a}))$ - these are areas with higher speckle contrast, in some cases caused by specular reflection due to accumulation of water. Applying groupwise registration reduced the artefacts in the contrast image (Fig. 4(b)) and improved flow contrast (SNR: 2.88 without registration vs. 2.97 with registration)

\section{Discussion}

Our results demonstrate the potential of endoscopic LSCI for imaging the fetal placental vasculature during intrauterine procedures. Our approach revealed the perfused vessels in high contrast relative to background both in the optical phantom and in the ex vivo human placenta, in air or under water, and in the presence of motion. Intralipid flow could be imaged down to a depth of $4 \mathrm{~mm}$ in the static phantom, $3 \mathrm{~mm}$ in the phantom under water, and $2 \mathrm{~mm}$ in the moving phantom. In the placenta, small branching capillaries could be clearly identified in the speckle contrast images but not as easily under white light illumination. 
(a)

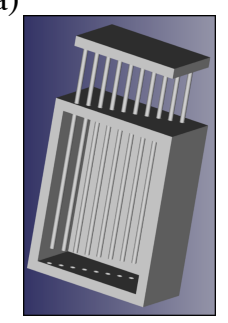

(b)

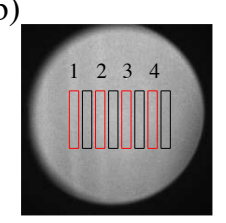

(d)

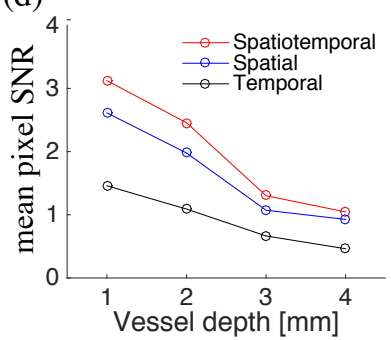

(c) depth $=1 \mathrm{~mm} \quad$ depth $=2 \mathrm{~mm} \quad$ depth $=3 \mathrm{~mm} \quad$ depth $=4 \mathrm{~mm}$

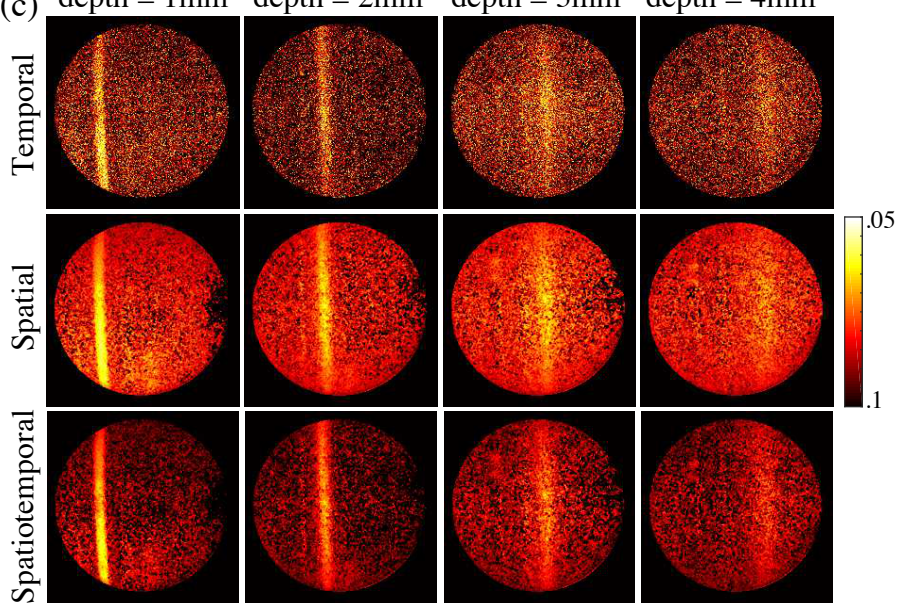

(e)

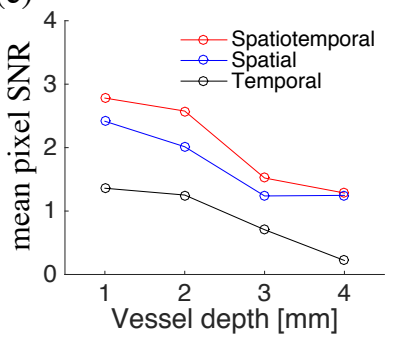

(f)

Fig. 2. (a) 3D-printed mould used for manufacturing the agar-based optical phantom. (b) Fetoscopic view of the phantom placed $1 \mathrm{~cm}$ from the fetoscope tip under white light illumination; vessels are filled with $1 \%$ intralipid. Numbers indicate the location and depth (in $\mathrm{mm}$ ) of the vessels, and red and black rectangles show the ROI and background region for each vessel. (c) Speckle contrast images of intralipid flow at variable vessel depths (columns) and obtained with different estimation algorithms (rows), with the same phantom position as in (b). (d-f) Signal to noise (SNR) analysis of contrast estimation algorithms in different setups: (d) phantom placed at $1 \mathrm{~cm}$ distance or (e) $2 \mathrm{~cm}$ distance; and (f) imaging in water with phantom at $1 \mathrm{~cm}$ distance.

Groupwise registration significantly improved the ability to correct for motion during image acquisition. Registering to the first frame, as proposed in $[5,6]$, may be adequate for near static conditions such as in neurosurgery, but may not be sufficiently robust for endoscopic LSCI. Our results showed that registration to the first frame failed to converge in several cases (Fig. 3), whereas groupwise registration had converged within the same number of iterations and using the same parameters. The next stage that we started implementing is to develop a real-time endoscopic LSCI system using a fast registration algorithm running on GPUs. Speckle contrast image registration can potentially be improved further by performing it simultaneously to endoscopic video registration, taking advan- 


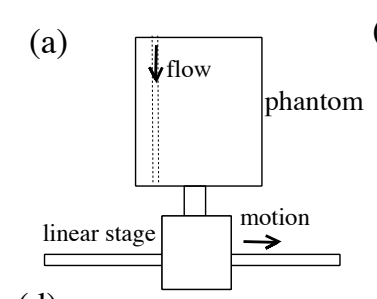

(d)

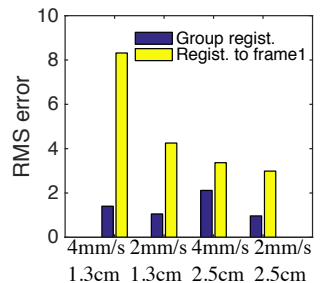

(e1) (b1) groupwise reg. (b2)

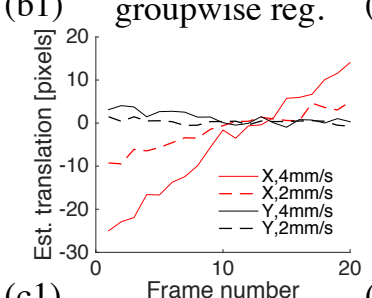

(c1)

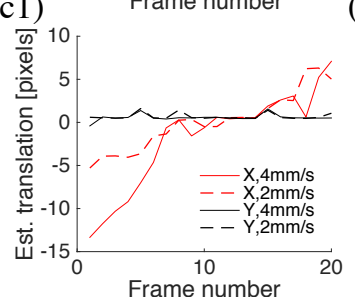

(e3) (b2) 1st frame reg.

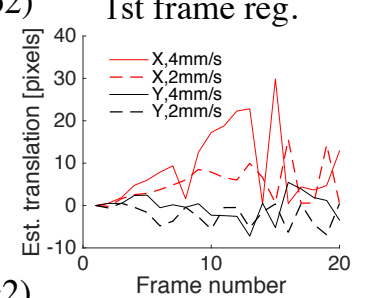

(c2)

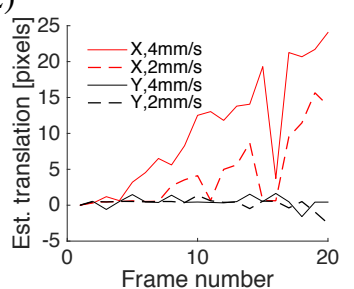

(e4)
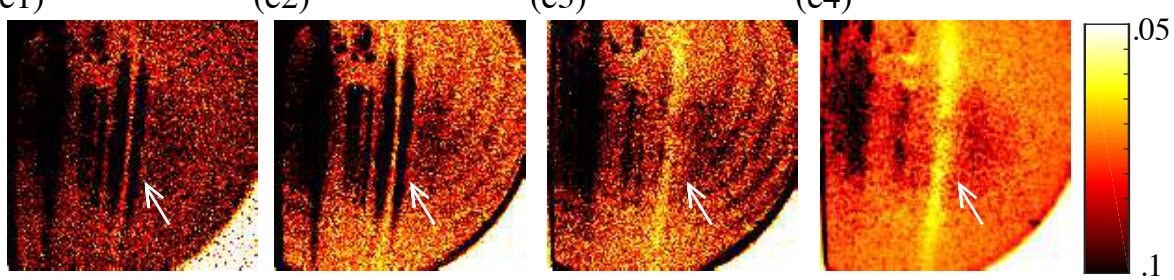

Fig. 3. (a) Setup for testing speckle contrast image registration. (b) Estimated X-Y translations for each frame using (b1) groupwise registration or (b2) registration to the 1st frame. Phantom was $1.3 \mathrm{~cm}$ away from fetoscope tip. (c) Same as in (b), but with phantom placed at $2.5 \mathrm{~cm}$ distance. (d) RMS error of estimated X displacements with respect to linear fits. (e) Speckle contrast images of vessel region (phantom distance: $1.3 \mathrm{~cm}$, speed: $4 \mathrm{~mm} / \mathrm{s}$ ) using different combinations of contrast estimation and registration algorithms: (e1) no registration, temporal estimation; (e2) registration to 1st frame, temporal estimation; (e3) groupwise registration, temporal estimation; and (e4) groupwise registration, spatiotemporal estimation. Arrow: perfused vessel.

tage of the richer texture information in the video images. Additionally, camera stabilisation using robotic surgical systems may help LSCI.

Endoscopic LSCI lends itself well to integration into current clinical workflow, as fibre-coupled lasers are already used in conjunction with imaging. Ultimately, we envisage that the same laser fibre could be used for two purposes. For instance, with a double-clad fibre, the single-mode core could deliver light for speckle imaging while the outer multi-mode cladding could deliver light for photocoagulation. Together with suitable spectral filters, this type of system could potentially be used to monitor photocoagulation in real-time. An integrated photocoagulation and endoscopic LSCI system would have great potential for use in intrauterine procedures, particularly for the TTTS procedure. Future work will focus on the development of this integrated system. 
(a)

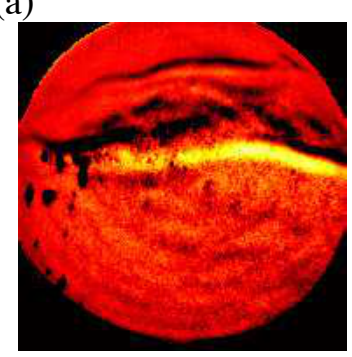

(b)

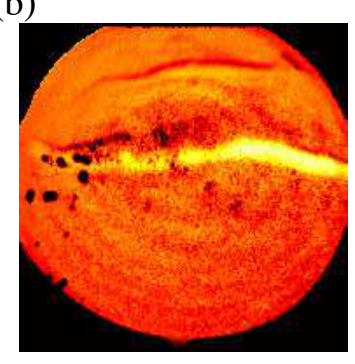

(c)

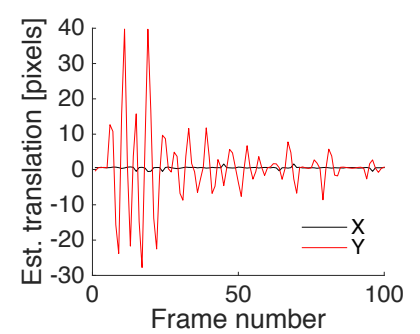

Fig. 4. Imaging placenta perfusion with motion. (a) Spatiotemporal contrast image of unregistered data (100 frames). (b) Spatiotemporal contrast estimation after groupwise registration, with fewer irregularities in background. (c) Estimated X-Y translation.

Acknowledgements. This work was supported through an Innovative Engineering for Health award by the Wellcome Trust [WT101957]; Engineering and Physical Sciences Research Council (EPSRC) [NS/A000027/1] and an NIHR BRC UCLH/UCL High Impact Initiative.

\section{References}

1. Hecht, N., Woitzik, J., König, S., Horn, P., Vajkoczy, P.: Laser speckle imaging allows real-time intraoperative blood flow assessment during neurosurgical procedures. Journal of Cerebral Blood Flow and Metabolism 33 (2013) 1000-7

2. Bray, R.C., Forrester, K.R., Reed, J., Leonard, C., Tulip, J.: Endoscopic laser speckle imaging of tissue blood flow: Applications in the human knee. Journal of Orthopaedic Research 24 (2006) 1650-1659

3. Senat, M.V., Deprest, J., Boulvain, M., Paupe, A., Winer, N., Ville, Y.: Endoscopic laser surgery versus serial amnioreduction for severe twin-to-twin transfusion syndrome. New England Journal of Medicine 351(2) (2004) 136-144

4. Slaghekke, Lewi, L., Middeldorp, J.M., Weingertner, A.S., Klumper, F.J., Dekoninck, P., Devlieger, R., Lanna, M.M., Deprest, J., Favre, R., Oepkes, D., Lopriore, E.: Residual anastomoses in twin-twin transfusion syndrome after laser: the Solomon randomized trial. American Journal of Obstetrics and Gyn. 211(3) (2014) 285-e1

5. Miao, P., Rege, A., Li, N., Thakor, N.V., Tong, S.: High resolution cerebral blood flow imaging by registered laser speckle contrast analysis. IEEE Transactions on Biomedical Engineering 57 (2010) 1152-1157

6. Richards, L.M., Towle, E.L., Fox, J.D.J., Dunn, A.K.: Intraoperative laser speckle contrast imaging with retrospective motion correction for quantitative assessment of cerebral blood flow. Neurophotonics 1(1) (2014) 15006

7. Boas, D.A., Dunn, A.K.: Laser speckle contrast imaging in biomedical optics (2010)

8. Guimond, A., Meunier, J., Thirion, J.P.: Average brain models: A convergence study. Computer vision and image understanding 77(2) (2000) 192-210

9. Klein, S., Staring, M., Murphy, K., Viergever, M.A., Pluim, J.P.: Elastix: a toolbox for intensity-based medical image registration. IEEE Transactions on Medical Imaging 29(1) (2010) 196-205 